\title{
Resting State BOLD Variability in Alzheimer's Disease: A Marker of Cognitive Decline or Cerebrovascular Status?
}

\section{OPEN ACCESS}

Edited by:

P. Hemachandra Reddy,

Texas Tech University Health Sciences Center, United States

Reviewed by:

Ravi Rajmohan,

Texas Tech University Health

Sciences Center, United States

Sunanda D. Mitra,

Texas Tech University, United States

*Correspondence: Jodie R. Gawryluk gawryluk@uvic.ca

${ }^{\dagger}$ Data used in preparation of this article were obtained from the Alzheimer's Disease Neuroimaging Initiative database (adni.loni.usc.edu). As such, the investigators within the $A D N /$ contributed to the design and implementation of $A D N I$ and/or provided data but did not participate in analysis or writing of this report. A complete listing of $A D N /$ investigators can be found at: $h$ ttp://adni./oni.usc. edu/wp-content/uploads/how_to apply/ADNI_Acknowledgement_ List.pdf.

Received: 10 December 2017 Accepted: 02 February 2018 Published: 21 February 2018

Citation:

Scarapicchia V, Mazerolle EL, Fisk JD, Ritchie LJ and Gawryluk JR (2018) Resting State BOLD Variability

in Alzheimer's Disease: A Marker of Cognitive Decline or

Cerebrovascular Status?

Front. Aging Neurosci. 10:39. doi: 10.3389/fnagi.2018.00039

\author{
Vanessa Scarapicchia ${ }^{1}$, Erin L. Mazerolle², John D. Fisk ${ }^{3,4,5,6,7}$, \\ Lesley J. Ritchie ${ }^{8}$ and Jodie R. Gawryluk ${ }^{1 *}$ for the Alzheimer's Disease \\ Neuroimaging Initiative ${ }^{\dagger}$
}

\begin{abstract}
${ }^{1}$ Department of Psychology, University of Victoria, Victoria, BC, Canada, ${ }^{2}$ Department of Radiology and The Hotchkiss Brain Institute, University of Calgary, Calgary, AB, Canada, ${ }^{3}$ Department of Psychology and Neuroscience, Dalhousie University, Halifax, NS, Canada, ${ }^{4}$ Department of Psychology, Nova Scotia Health Authority, Queen Elizabeth II Health Sciences Centre, Halifax, NS, Canada, ${ }^{5}$ Department of Psychiatry, Dalhousie University, Halifax, NS, Canada, ${ }^{6}$ Department of Psychology and Neuroscience, Dalhousie University, Halifax, NS, Canada, ${ }^{7}$ Department of Medicine, Dalhousie University, Halifax, NS, Canada, ${ }^{8}$ Department of Clinical Health Psychology, University of Manitoba, Winnipeg, MB, Canada
\end{abstract}

Background: Alzheimer's disease (AD) is a neurodegenerative disorder that may benefit from early diagnosis and intervention. Therefore, there is a need to identify early biomarkers of $A D$ using non-invasive techniques such as functional magnetic resonance imaging (fMRI). Recently, novel approaches to the analysis of resting-state fMRI data have been developed that focus on the moment-to-moment variability in the blood oxygen level dependent (BOLD) signal. The objective of the current study was to investigate BOLD variability as a novel early biomarker of $A D$ and its associated psychophysiological correlates.

Method: Data were obtained from the Alzheimer's Disease Neuroimaging Initiative (ADNI) 2 database from 19 participants with $A D$ and 19 similarly aged controls. For each participant, a map of BOLD signal variability $\left(\mathrm{SD}_{B O L D}\right)$ was computed as the standard deviation of the BOLD timeseries at each voxel. Group comparisons were performed to examine global differences in resting state $\mathrm{SD}_{\mathrm{BO} D}$ in $\mathrm{AD}$ versus healthy controls. Correlations were then examined between participant $\mathrm{SD}_{\mathrm{BO}}$ maps and (1) ADNI-derived composite scores of memory and executive function and (2) neuroimaging markers of cerebrovascular status.

Results: Between-group comparisons revealed significant $(p<0.05)$ increases in $\mathrm{SD}_{\mathrm{BOLD}}$ in patients with $\mathrm{AD}$ relative to healthy controls in right-lateralized frontal regions. Lower memory scores and higher WMH burden were associated with greater $\mathrm{SD}_{\mathrm{BO}}$. in the healthy control group $(p<0.1)$, but not individuals with AD.

Conclusion: The current study provides proof of concept of a novel resting state $\mathrm{fMRI}$ analysis technique that is non-invasive, easily accessible, and clinically compatible. To further explore the potential of $\mathrm{SD}_{\mathrm{BO}}$ D as a biomarker of $\mathrm{AD}$, additional studies in larger, longitudinal samples are needed to better understand the changes in $\mathrm{SD}_{\mathrm{BOLD}}$ that characterize earlier stages of disease progression and their underlying psychophysiological correlates.

Keywords: signal variability, Alzheimer's disease, biomarker, BOLD fMRI, aging 


\section{INTRODUCTION}

Alzheimer's disease is a progressive, neurocognitive disorder characterized by impairments in memory, as well as other cognitive domains, including language, visuospatial skills, and executive functions (Alzheimer's Association, 2016). Although a number of factors have been associated with the development of $\mathrm{AD}$, epidemiological evidence suggests that the strongest risk factor for $\mathrm{AD}$ is age. Nearly one out of every nine individuals beyond the age of 65 is expected to develop the disease; by age 85 , this figure rises markedly to an estimate of nearly one out of three (Alzheimer's Association, 2016). In light of globally increasing life expectancies and a rapidly aging population, $\mathrm{AD}$ has become an urgent public health concern (Winblad et al., 2016).

At present, there are no curative treatments for $\mathrm{AD}$ (Scheltens et al., 2016). Available treatment options are limited and focus primarily on delaying the progression of symptoms (Wilkinson, 2012; Alzheimer's Association, 2016). There are a number of lifestyle factors, both reversible and irreversible, that may increase or reduce an individual's risk for developing dementia. It has recently been proposed that up to a third of all cases of dementia may theoretically be prevented through populationlevel interventions targeted at modifiable risk factors (Livingston et al., 2017). While there is limited evidence to support the use of mass cognitive screens due to their unclear benefits, improving the identification of high-risk groups will be imperative in order to deliver targeted treatment. This points to an everincreasing need for the identification of reliable, early biomarkers for $\mathrm{AD}$.

The ideal technique for biomarker identification would be non-invasive, easily repeatable, and widely available, as is MRI. Although most MRI based biomarker research on $\mathrm{AD}$ to date has focused on structural changes in gray matter (GM; Cash et al., 2014), MRI can also provide relevant measures of brain function. BOLD functional MRI (fMRI) is a MRI based technique that allows for non-invasive examination of brain function by measuring fluctuations in signal intensity over time that are a consequence of oxygenated blood supplying active neurons. Recently, resting-state fMRI (rsfMRI) has emerged as a promising clinical imaging method, as it eliminates the cognitive burden of task performance that is characteristic of task-based fMRI and thus reduces the level of compliance required of the patient (Fox and Greicius, 2010; Mueller et al., 2012).

Traditionally, the majority of fMRI investigations have based their findings on patterns of mean brain activity. This is based on the longstanding premise that the mean value across an fMRI time-series represents the average, and therefore most representative, "signal" among a distribution of unwanted "noise" (Garrett et al., 2010). This stands in contrast to theories postulating that the brain is an intrinsically variable system, and that such variability may provide meaningful insights into its functional architecture (Stein et al., 2005; Faisal et al., 2008; Deco

Abbreviations: AD, Alzheimer's disease; ADNI, Alzheimer's Disease Neuroimaging Initiative; BOLD, blood oxygen level dependent; FSL, Functional MRI of the Brain Software Library; MRI, magnetic resonance imaging; MMSE, Mini Mental Status Exam; $\mathrm{SD}_{\mathrm{BOLD}}$, standard deviation of the fMRI BOLD signal; WMH, white matter hyperintensity; WMS, Wechsler Memory Scale. et al., 2011). Stemming from these emerging conceptualizations, novel approaches to analyzing rsfMRI data have been developed that focus on the moment-to-moment variability in the BOLD signal (Garrett et al., 2013b).

In recent years, an increasing number of studies have focused on BOLD signal variability in normative aging. For instance, a study by Garrett et al. (2010) examined the BOLD signal standard deviation ( $\mathrm{SD}_{\mathrm{BOLD}}$ ) in a sample of healthy adults ranging in age from 20 to 85 and found that patterns of resting-state $\mathrm{SD}_{\mathrm{BOLD}}$ were generally more variable in younger adults. They suggested that this finding may reflect reductions in synaptic complexity and integrity in older age (Garrett et al., 2010). In support of this framework, subsequent task-based fMRI studies by the same group found that greater BOLD signal variability was associated with younger age and superior cognitive task performance (Garrett et al., 2011, 2013a, 2014; Grady and Garrett, 2014). Other studies have found greater resting-state $\mathrm{SD}_{\mathrm{BOLD}}$ to be associated with increased microstructural integrity of white matter (WM) pathways in healthy older adults (Burzynska et al., 2015a,b). This relationship has not been a consistent finding, however, and some studies have regional increases in fMRI BOLD variance in older versus younger healthy adults (Garrett et al., 2010, 2011; Nomi et al., 2017), as well in individuals with stroke (Kielar et al., 2016), multiple sclerosis (Petracca et al., 2017) and other neurological disorders (Zöller et al., 2017). Thus, while the source of increased regional BOLD fluctuations remains unclear, it is conceivable that this may reflect sub-optimal functioning and/or compensatory mechanisms (Garrett et al., 2010; Nomi et al., 2017; Petracca et al., 2017). In general, the emerging consensus appears to be that variability in the rsfMRI BOLD signal may be a viable index of age-related cognitive decline that could provide new insights into age-related pathologies. Some rsfMRI studies have begun to examine other aspects of the temporal dynamics of spontaneous BOLD fluctuations in mild cognitive impairment (Han et al., 2011; Xi et al., 2012; Zhao et al., 2015) and AD (Liu et al., 2013, 2014), but the utility of variance measures such as $\mathrm{SD}_{\mathrm{BOLD}}$ as a biomarker of $\mathrm{AD}$ requires further study.

Recently, Makedonov et al. (2013) used a slightly different measure of variability and found that BOLD fluctuations in WM regions are higher in (1) healthy older adults relative to younger adults, and (2) in the normal appearing WM structures of older adults with cerebral small vessel disease relative to healthy older adults (CSVD; Makedonov et al., 2013). The authors argued that increased arterial stiffness caused by cerebrovascular disease may result in greater pulsatility in vascular networks and small vessels, and result in the increased temporal variance of the BOLD signal observed in their study. Support for this suggestion was provided by a subsequent study that found greater variation of spontaneous BOLD fluctuations in both GM and WM in hypertensive elderly patients (Jahanian et al., 2014). Together, these findings suggest that resting-state BOLD variance may serve as a physiological signal related to an individual's cerebrovascular status. Most recently, Makedonov et al. (2016) examined resting state in WM in individuals with $\mathrm{AD}$ and found significantly increased BOLD fluctuations in those with AD relative to those with mild cognitive impairment and to age-matched controls. Furthermore, 
they found that the increased BOLD fluctuations had a negative relationship with memory scores, thereby supporting a link between increased WM BOLD fluctuations and lower cognitive function.

The possibility that differences in BOLD fluctuations may reflect underlying differences in cerebrovascular health not captured by existing $\mathrm{AD}$ biomarkers that are associated with cognitive functioning, clearly warrants further study. To this end, we aimed to (1) examine whole brain differences in $\mathrm{SD}_{\mathrm{BOLD}}$ in a group of individuals with $\mathrm{AD}$ and healthy age-matched controls, (2) determine whether measures of BOLD variability were related to measures of cognitive ability, and (3) investigate the relations between BOLD variability and WM cerebrovascular dysfunction. We hypothesized that there would be (1) widespread differences in rsfMRI BOLD variance in patients with $A D$ versus healthy controls, (2) a relation between BOLD variability and participant cognitive test performance, and (3) a positive relation between BOLD variability and MRI-based measures of WM lesion burden.

\section{MATERIALS AND METHODS}

\section{ADNI Database}

All data for the present study were obtained from the Alzheimer's Disease Neuroimaging Initiative 2 (ADNI-2) database ${ }^{1}$. The ADNI, led by principal investigator Michael W. Weiner, began in 2003 as a partnership between the National Institute on Aging, the National Institute on Biomedical Imaging and Engineering, the Food and Drug Administration, as well as other private and public non-profit organizations. Since its launch, the primary goal of ADNI has been to develop more sensitive methods that may be able to detect $\mathrm{AD}$ at its earliest time point, in order to maximize the efficacy of future disease modifying or delaying interventions. Now in its fourth phase, the ADNI is focused on tracking the longitudinal progression of neuroimaging, laboratory, and neuropsychological $\mathrm{AD}$ biomarkers in participants from acquisition sites across Canada and the United States. For further information, please $\operatorname{see}^{2}$.

\section{Participants}

All participants were selected from the ADNI-2 database, as the ADNI-1 phase did not collect rsfMRI data. In order to ensure the best variable control possible, inclusion to the $\mathrm{AD}$ group required (1) a clinician-confirmed diagnosis of mild AD at the screening visit, (2) complete rsfMRI data available for the first time point of the study. Given that ADNI participants are classified into groups (AD or "normal") based on their clinical presentation at the screening visit, participants were selected from the first available time point ( $\sim 14$ days post-screening) to ensure the continued accuracy of this diagnosis. The ADNI2 database ultimately contained a total of $34 \mathrm{AD}$ patients with rsfMRI data that met these criteria. Due to the novel methods used in the current study, extra caution was taken to ensure that

${ }^{1} \mathrm{http}: / /$ adni.loni.usc.edu

${ }^{2} \mathrm{http}: / /$ www.adni-info.org
TABLE 1 | Participant demographics.

\begin{tabular}{lccc}
\hline & AD & CN & AD vs. CN \\
\hline Age & $72.7 \pm 6.5$ & $74.7 \pm 6.9$ & $p=0.365$ \\
Females & 12 & 11 & $p=0.740$ \\
Males & 7 & 8 & \\
Education (years) & $16.2 \pm 2.6$ & $16.3 \pm 2.3$ & $p=0.896$
\end{tabular}

the results of the analysis were not confounded by error-related noise: $\mathrm{AD}$ participants from this total pool were then manually selected for the current study based on a rigorous screening of raw data quality, as well as the accuracy of registration of the patient's functional and structural images to standard stereotactic space, resulting in a final total of 19 participants in the $\mathrm{AD}$ group. An equivalent number of healthy control participants were then selected to form a comparable group. In the end, data were obtained from the first available time point from 19 individuals with $\mathrm{AD}$ (mean age $=72.7$ years, $S D=6.5 ; 12$ females) and 19 healthy age-matched controls (mean age $=74.7$ years, $S D=6.9 ; 11$ females). No significant differences were found between groups in participant age, sex, or education level. Participant demographic information can be found in Table $\mathbf{1}$.

Diagnostic classification of $\mathrm{AD}$ participants was made by ADNI investigators according to diagnostic criteria for Probable $\mathrm{AD}$ established by the National Institute of Neurological and Communicative Disorders and Stroke and the Alzheimer's Disease Related Disorders Association (NINCDS-ADRA; McKhann et al., 1984). Participants in the AD cohort also exhibited abnormal memory function on the Logical Memory II subscale of the revised Wechsler Memory Scale (WMS II, $\leq 8$ for 16 years education and above), a Mini Mental State Exam (MMSE) between 20 and 26 (inclusive), and a Clinical Dementia Rating of 0.5 (very mild) or 1 (mild).

All control participants were free of memory complaints and deemed cognitively normal based on clinical assessments by the site physician showing an absence of significant impairment in cognitive functioning and performance of daily activities. Participants in the control cohort also exhibited normal memory function on the Logical Memory II subscale of the revised WMS (WMS II, $\geq 9$ for 16 years of education and above), a MMSE score between 24 and 30 (inclusive), and a Clinical Dementia Rating of 0 . For more information on group classifications, including all additional eligibility criteria, please consult the ADNI-2 procedures manual (Alzheimer's Disease Neuroimaging Initiative, 2008).

All ADNI participants or their authorized representatives provided written informed consent approved by the Institutional Review Board at each acquisition site. For the purpose of the current study, secondary use of the data was approved by the Human Research Ethics Board at the University of Victoria (Victoria, BC, Canada).

\section{Image Acquisition}

MRI data were downloaded with permission from the ADNI. All images were acquired on 3.0 Tesla Philips MRI scanners across 10 
North American acquisitions sites according to the standardized ADNI protocol (Jack et al., 2008). Whole-brain anatomical MRI scans were acquired sagittally, with a T1-weighted MPRAGE sequence, with the following parameters: $1.2 \mathrm{~mm}$ slice thickness, $256 \times 256 \times 170$ acquisition matrix, echo time (TE) of $3 \mathrm{~ms}$, in-plane voxel dimension of $1 \mathrm{~mm}^{2}$, repetition time (TR) of $7 \mathrm{~ms}$, and flip angle of $9^{\circ}$. Functional MRI scans were obtained during resting state; participants were instructed to lay quietly in the scanner with their eyes open. Resting state fMRI scans were obtained with a $\mathrm{T} 2 *$-weighted echo-planar imaging sequence with the following parameters: 140 volumes, $64 \times 64 \times 48$ acquisition matrix (voxel size $=3.3 \mathrm{~mm}^{3}$ ), TE of $30 \mathrm{~ms}$, TR of $3000 \mathrm{~ms}$, and flip angle of $80^{\circ}$.

The participant's T2-weighted fluid-attenuated inversion recovery (FLAIR) images were obtained for the purpose of lesion volume computation. The T2-weighted FLAIR images were obtained with $5.0 \mathrm{~mm}$ axial slices, a $256 \times 256 \times 35$ acquisition matrix, a TE of $90 \mathrm{~ms}$, an inversion time of $2500 \mathrm{~ms}$, an in-plane voxel dimension of $0.85938 \mathrm{~mm}^{2}$, a TR of $9000 \mathrm{~ms}$, and a flip angle of $90^{\circ}$.

\section{Data Analysis}

\section{Image Preprocessing}

All analysis steps were performed using tools within the FSL version 5.0 (Analysis Group, FMRIB, Oxford, United Kingdom³ Smith et al., 2004). Non-brain tissue in the raw T1 images was removed using the automated Brain Extraction Tool (Smith, 2002), followed by manual verification and optimization for each subject. BOLD data preprocessing was performed in FSL's FEAT as follows: each functional image was motion corrected and registered to their high-resolution T1 structural image that was linearly registered to standard stereotaxic space using a $12^{\circ}$ of freedom transformation. A non-linear registration of the structural image to standard stereotactic space was also applied to account for potential local deformations in brains of the patient group. Additionally, global signal regression (GSR) was performed to correct for confounding physiological noise and to improve the detection of localized variation in the BOLD signal (Desjardins et al., 2001; Macey et al., 2004; Fox et al., 2009).

\section{Statistical Comparisons}

Individuals with $\mathrm{AD}$ were compared to age-matched controls in the group-level analysis. All results were examined at a $p<0.05$ significance level, unless otherwise stated.

\section{Resting-State BOLD Variability $\left(\mathrm{SD}_{\mathrm{BO}}\right)$}

Though different conceptualizations of brain signal variability exist, an increasingly popular approach in rsfMRI involves examining the distributional width of the neuroimaging timeseries by computing the signal variance or standard deviation ( $\mathrm{SD}_{\mathrm{BOLD}}$ ) across voxels (for a review, see Garrett et al., 2013b). Following this framework, the current study derived a measure of BOLD variability by first obtaining the variance of the residual signal left over after preprocessing at each voxel across the whole brain. The square root of the variance within each

${ }^{3}$ http://fsl.fmrib.ox.ac.uk voxel was subsequently computed in order to arrive at a $\mathrm{SD}_{\mathrm{BOLD}}$ map for each participant; this map effectively describes the standard deviation of the BOLD timeseries at each voxel within both GM and WM regions. The derived $\mathrm{SD}_{\mathrm{BOLD}}$ maps from each individual were then merged into a single $4 \mathrm{D}$ file and smoothed with a Gaussian kernel (6 $\mathrm{mm})$. To examine differences in resting state $\mathrm{BOLD}$ variability in $\mathrm{AD}$ patients versus healthy age-matched controls, between-group contrast comparisons of $\mathrm{SD}_{\mathrm{BOLD}}$ were performed using randomize (Winkler et al., 2014) with threshold-free cluster enhancement and correction for multiple comparisons.

\section{Relations between BOLD Variability and Cognitive Scores (Executive Function and Memory)}

Neuropsychological data were obtained according to the protocol outlined in the ADNI-2 procedures manual (Alzheimer's Disease Neuroimaging Initiative, 2008). The ADNI-2 database contains neuropsychological test scores for individual tests, as well as composite scores for the examination of memory and executive function. Deficits in both memory and executive function are core clinical characteristics of $\mathrm{AD}$ that have been shown to occur in the early stages of the disease (Baudic et al., 2006; Sperling et al., 2010). The use of composite measures, as opposed to individual cognitive tests, has been suggested to increase measurement precision and further limit the challenges associated with multiple hypothesis testing (Crane et al., 2012; Gibbons et al., 2012). In order to determine whether differences in resting state BOLD variability were related to participant cognitive test scores, correlations were examined between the participant's $\mathrm{SD}_{\mathrm{BOLD}}$ maps, as described above, and composite clinical scores for (1) memory performance (ADNI-MEM) and (2) executive function (ADNI-EF). Both of these cognitive measures have been derived from the ADNI neuropsychological test battery using item response theory (IRT) methods and validated in subsequent studies. Specifically, the ADNI-MEM score was derived from a single-factor confirmatory factor analysis model performed by Crane et al. (2012) using data from the Rey Auditory Verbal Learning Test (RAVLT), AD Assessment Schedule - Cognition (ADAS-Cog), MMSE, and the WMS Logical Memory subscale. The ADNI-EF score was derived by Gibbons et al. (2012) using a bi-factor confirmatory factor analysis model with data from the Wechsler Adult Intelligence Scale - Revised (WAIS-R) Digit Symbol Substitution, Digit Span Backward, Trails A and B, Category Fluency, and Clock Drawing. Due to missing data, two participants from each of the $\mathrm{AD}$ and $\mathrm{CN}$ groups were omitted from this analysis. Statistical computations were performed using randomize with threshold-free cluster enhancement and correction for multiple comparisons. Information on group cognitive performance can be found in Table 2 .

TABLE 2 | Participant clinical scores (separated by group).

\begin{tabular}{lccc}
\hline & AD & CN & AD vs. CN \\
\hline ADNI-MEM & $-0.9516 \pm 0.5250$ & $0.8270 \pm 0.4996$ & $p=0.000$ \\
ADNI-EF & $-0.8996 \pm 0.6935$ & $0.7224 \pm 0.4510$ & $p=0.000$
\end{tabular}




\section{Relations between BOLD Variability and Cerebrovascular Status (WM Lesion Volumes)}

To derive a measure of cerebrovascular status, we identified participant white matter hyperintensities (WMHs) on FLAIR images and determined total WM lesion burden for each participant. Following data screening, four AD participants were omitted from this analysis due to FLAIR image motion artifact. Lesions were segmented by the lesion prediction algorithm (LPA; Schmidt, 2017), using FLAIR images only, as implemented in the LST toolbox version $2.0 .15^{4}$ for the Statistical Parametric Mapping (SPM) software. Briefly, the LPA is a binary classifier in the form of a logistic regression model that was initially trained on the data of 53 multiple sclerosis patients with severe lesion patterns (Schmidt et al., 2012). Parameters of this model are used to segment lesions in new images by computing an estimate for the lesion probability within each voxel. In the current analysis, lesion volumes $(\mathrm{ml})$ derived from each participant's probabilistic map were extracted to derive a total lesion volume for each subject. To account for variability in parenchymal volume across participants, total brain volumes for each participant were computed using FMRIB's Automated Segmentation Tool (FAST; Zhang et al., 2001). Lesion volumes were subsequently converted to a value representing the fraction of total brain volume (TBV) occupied by WMHs in each participant. Correlations were then examined between these values and the participants' restingstate $\mathrm{SD}_{\mathrm{BOLD}}$ maps. As in previous steps, statistical computations were performed using randomize with threshold-free cluster enhancement and correction for multiple comparisons.

\section{Relations between WMH Burden and Cognitive Performance}

As a secondary post hoc analysis, the relation between cognitive performance (ADNI-MEM and ADNI-EF) and WMH burden was examined across groups to better understand the contributions of cerebrovascular burden to the overall sample. Partial correlation coefficients were examined with two-tailed significance $(p<0.05)$, controlling for both age and education.

\section{RESULTS}

\section{Differences in Resting State $\mathrm{SD}_{\mathrm{BO}}$ in Patients with $A D$ versus Healthy Controls}

Between-group comparisons were performed to examine differences in resting-state $\mathrm{SD}_{\mathrm{BOLD}}$ in participants with $\mathrm{AD}$ relative to healthy age-matched controls. The results of this analysis revealed significant differences in BOLD variability ( $p<0.05$, corrected for multiple comparisons) in righthemispheric GM and WM regions (Table 3). Specifically, $\mathrm{AD}>\mathrm{CN}$ contrasts showed a greater resting state $\mathrm{SD}_{\mathrm{BOLD}}$ in the $\mathrm{AD}$ group, predominantly in the right superior frontal gyrus and adjacent frontal regions, including the right precentral gyrus and right putamen. Significant WM regions were similarly right-lateralized and included portions of the right superior longitudinal fasciculus (frontal and temporal components) and

\footnotetext{
${ }^{4}$ http://www.statistical-modelling.de/lst.html
}

TABLE 3 | Brain regions showing increased $\mathrm{SD}_{\mathrm{BOLD}}$ in participants with $\mathrm{AD}$ relative to healthy controls ( $p<0.05$, corrected for multiple comparisons).

\begin{tabular}{lcccc}
\hline Brain region & Hemisphere & \multicolumn{3}{c}{ MNI coordinates } \\
\cline { 3 - 5 } & & $\mathbf{x}$ & $\mathbf{y}$ & $\mathbf{z}$ \\
\hline Gray matter & & & & \\
Superior frontal gyrus & $\mathrm{R}$ & 24 & 0 & 68 \\
Precentral gyrus & $\mathrm{R}$ & 56 & 4 & 32 \\
Putamen & $\mathrm{R}$ & 32 & -8 & 0 \\
White matter & & & & \\
Superior longitudinal fasciculus & $\mathrm{R}$ & 36 & -22 & 28 \\
Posterior corona radiata & $\mathrm{R}$ & 24 & -32 & 36 \\
Superior corona radiata & $\mathrm{R}$ & 24 & -6 & 38 \\
Anterior corona radiata & $\mathrm{R}$ & 20 & 24 & 24 \\
Forceps minor & $\mathrm{R}$ & 20 & 38 & 16 \\
External capsule & $\mathrm{R}$ & 30 & -8 & 12 \\
\hline
\end{tabular}

Coordinates are shown in Montreal Neurological Institute (MNI) standard stereotaxic space.

right superior and inferior corona radiata (Figure 1). No GM or $\mathrm{WM}$ regions were found to have increased $\mathrm{SD}_{\mathrm{BOLD}}$ in healthy controls relative to individuals with AD.

\section{Relationship between Resting State $S_{D_{B O L D}}$ and Cognitive Scores ADNI-MEM}

When a conventional significance threshold $(p<0.05)$ was employed, no significant relationship was found between ADNIMEM scores and $\mathrm{SD}_{\mathrm{BOLD}}$ in either participants with $\mathrm{AD}$ or healthy controls. However, given the novelty and exploratory nature of this research, relationships were also explored using a more liberal threshold $(p<0.1$, corrected for multiple comparisons). In doing so, an association was identified in the healthy control group, who demonstrated a negative relationship between $\mathrm{SD}_{\mathrm{BOLD}}$ and ADNI-MEM scores in both GM and WM regions (Table 4). Specifically, lower ADNI-MEM scores were associated with greater $\mathrm{SD}_{\mathrm{BOLD}}$ in the healthy control group in the left medial temporal lobe (MTL) and adjacent structures, including the right hippocampus and right amygdala, extending to the parahippocampal gyri bilaterally, as well as in the left superior longitudinal fasciculus and right inferior longitudinal fasciculus (Figure 2).

\section{ADNI-EF}

No significant relationship was found between ADNI-EF scores and $\mathrm{SD}_{\mathrm{BOLD}}$ in $\mathrm{AD}$ patients or healthy controls using either conventional or more liberal thresholds.

\section{Relations between Resting State $\mathrm{SD}_{\mathrm{BO}}$ and Participant WMH Volume}

Figure 3 shows the probabilistic lesion volume maps for a prototypical $\mathrm{AD}$ and healthy control participant. To account for total brain volume, lesion volumes were subsequently converted to a value representing the fraction of TBV occupied by $\mathrm{WMH}$ in each participant. On average, AD patients had higher total WM 


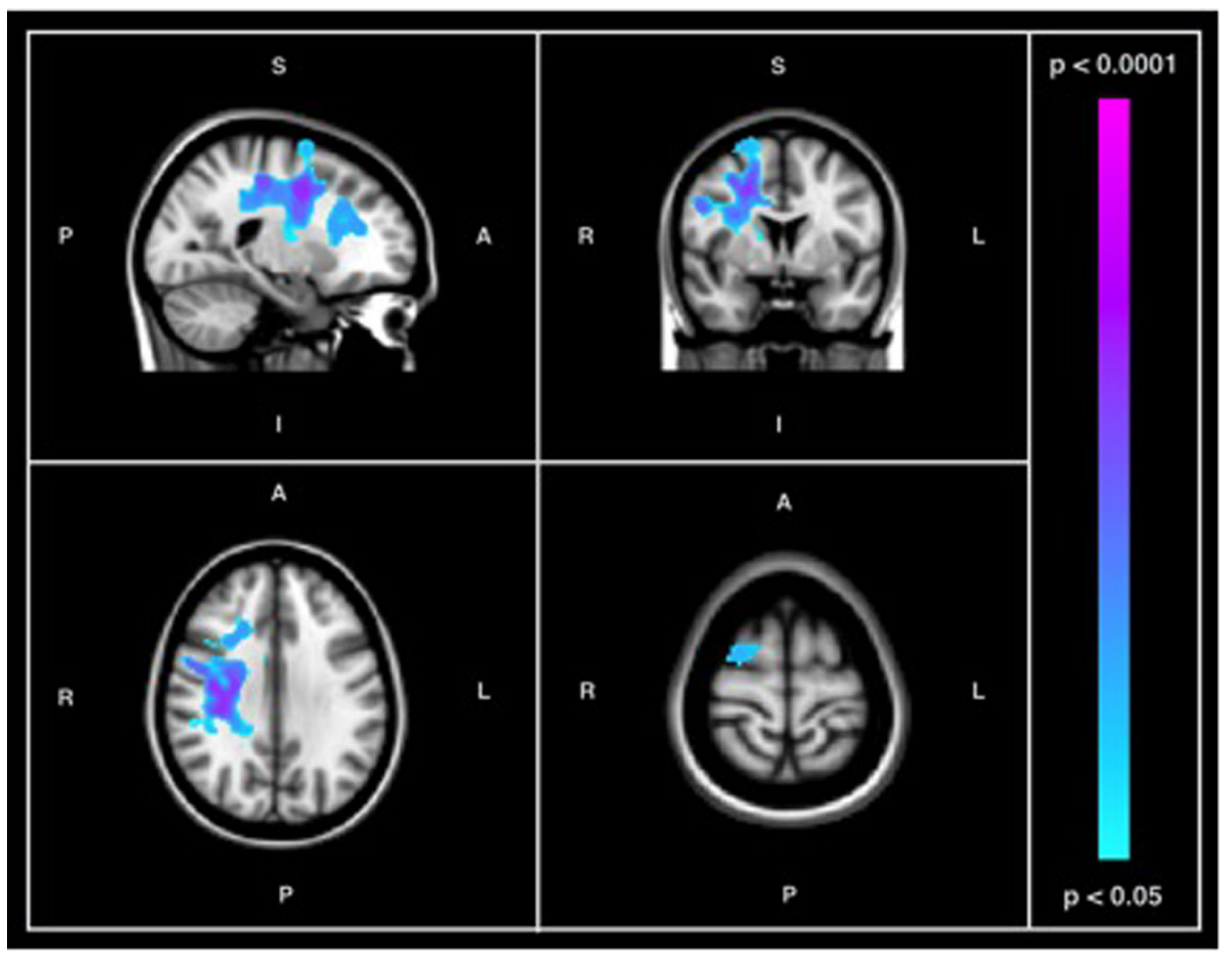

FIGURE 1 | Results of between-group comparison of $\mathrm{SD}_{B O L D}$ in participants with $\mathrm{AD}$ versus healthy controls showing regions of increased signal variability in individuals with $\mathrm{AD}(\mathrm{p}<0.05$, corrected for multiple comparisons) in both GM and WM. Images on overlaid on T1-weighted MNI152_T1_2mm standard template provided by the Functional MRI of the Brain's Software Library.

lesion burden (fractional lesion load $=0.011 \pm 0.007$ ) than agematched controls (fractional lesion load $=0.007 \pm 0.007$ ). Using a conventional significance threshold $(p<0.05)$, no significant relation was found between total $\mathrm{WMH}$ burden and $\mathrm{SD}_{\mathrm{BOLD}}$ in either group. However, using a more liberal threshold $(p<0.1$, corrected for multiple comparisons) a relationship was identified in the healthy control group, which showed a positive association between WHM lesion burden and $\mathrm{SD}_{\mathrm{BOLD}}$ in highly localized GM and WM regions (Table 5). Specifically, higher WMH lesion burden was associated with greater $\mathrm{SD}_{\mathrm{BOLD}}$ in temporal, frontoparietal, and orbitofrontal regions, and most prominently in the right parahippocampal gyrus (Figure 4).

\section{Relations between WMH Burden and Cognitive Performance Across Groups}

A significant negative correlation $(r=-0.462, p<0.01)$ was found between ADNI-MEM and WHM burden across participants, after correcting for age and education. Similarly, a significant negative relationship $(r=-0.482, p<0.05)$ was also found between ADNI-EF and WHM burden across participants, after correcting for age and education (Table 6).

\section{DISCUSSION}

The first goal of the current study was to examine and characterize differences in resting-state BOLD variability in
$\mathrm{AD}$ relative to normative aging. In light of previous studies reporting a robust association between regional BOLD variability and age-related cognitive decline (Garrett et al., 2010, 2011, 2013a), we anticipated widespread differences in resting-state BOLD variability in patients with $\mathrm{AD}$ relative to healthy older adults. We observed significant increases in $\mathrm{SD}_{\mathrm{BOLD}}$ in patients with $\mathrm{AD}$ in a number of $\mathrm{GM}$ and $\mathrm{WM}$ frontal regions, including portions of the superior frontal and precentral gyri, the superior longitudinal fasciculus, and widespread regions of the corona radiata (Table 3). Notably, both GM and WM regions with increased BOLD variability in $A D$ were right lateralized (Figure 1). No brain regions were found to have increased $\mathrm{SD}_{\mathrm{BOLD}}$ in healthy aging relative to AD.

The finding of increased BOLD variability in $\mathrm{AD}$ conflicts with earlier studies of $\mathrm{SD}_{\mathrm{BOLD}}$ in normative aging, wherein greater BOLD variability has been posited to serve as a neural marker of optimal brain function (Grady and Garrett, 2014). Given the neurodegeneration inherent in $\mathrm{AD}$, it is plausible to expect BOLD variability, when conceptualized as a neuronal signal of functional integrity, to be reduced in this population. However, studies have also revealed isolated regions of increased BOLD variability in older age (Garrett et al., 2010, 2011; Nomi et al., 2017), in stroke patients (Kielar et al., 2016) and in individuals with neurological disease (Petracca et al., 2017; Zöller et al., 2017), thereby alluding to a greater complexity the association between BOLD variability 
TABLE 4 | Brain regions showing a negative association between $\mathrm{SD}_{B O L D}$ and ADNI-MEM scores in the healthy control group $(p<0.1$, corrected for multiple comparisons).

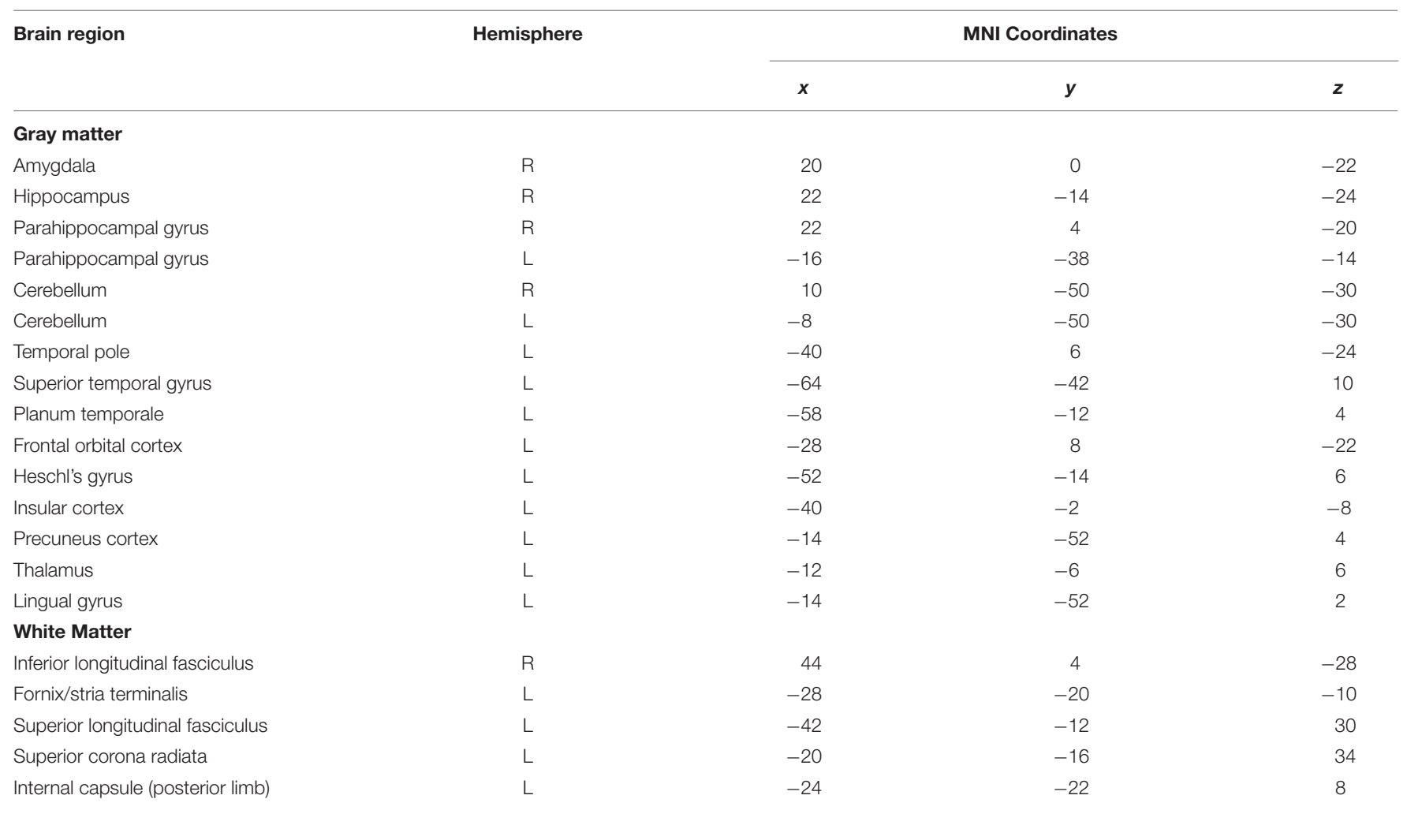

Coordinates are shown in Montreal Neurological Institute (MNI) standard stereotaxic space.

and functional integrity on a region-by-region basis. Notably, Makedonov et al. (2016), found that resting state BOLD fluctuations in WM were significantly increased in patients with $\mathrm{AD}$ relative to both participants with MCI and healthy controls. As a whole, it would appear that many of the studies in which increased BOLD fluctuations have been identified have examined patient populations. Given that brain signal variability has been found to exhibit robust agerelated developmental trajectories (e.g., Grady and Garrett, 2014), it is therefore possible that aberrant findings in patient groups may reflect a disruption of normative brain processes.

However, a potential barrier to reconciling the current results with those found in previous studies, including the seminal studies by Garrett et al. $(2010,2011)$, relates to the lack of methodological standardization in this area of research. Indeed, a review of the recent literature shows that a number of different variations of 'BOLD variability' measures have been employed in previous studies, with different methodologies used by different groups to derive what are ostensibly the same variance measures, including $\mathrm{SD}_{\mathrm{BOLD}}$. Though one of the aims of the current study was to increase transparency of the methodology used, this nonetheless remains a potential barrier to cross-study comparisons.

Another potential explanation for the current findings relates to the well-documented phenomenon of neural compensation in aging. In accordance with the present findings, many of the regions where increased BOLD variability has been identified in older or clinical populations have included portions of the frontal cortex (Petracca et al., 2017; Zöller et al., 2017), and particularly the superior frontal gyri (Garrett et al., 2010; Kielar et al., 2016). Interestingly, this localization to frontal regions is also reflected in mean-BOLD fMRI studies of aging, wherein prefrontal over-activation in older relative to younger adults has been postulated to reflect the additional recruitment of executive resources in support of memory maintenance (Reuter-Lorenz and Park, 2010). It is also notable that the right-hemispheric lateralization observed in the current study is also congruent with the existing BOLD fMRI compensatory literature: as summarized by Gauthier et al. (2013), one of the most common findings in aging is a decreased lateralization of the BOLD response, characterized primarily by a decrease in left frontal BOLD responses but an increase in the right frontal BOLD response (for a review see Reuter-Lorenz and Park, 2010). Extending these findings to the current study, it is therefore possible that similar mechanisms would apply in the resting-state, with the AD patients posing as an "accelerated aging" group relative to healthy aging. Keeping in mind the comparative-contrast methods in fMRI, this decreased frontal lateralization of in the AD group could plausibly have translated into an increased right frontal BOLD response when examining contrasts at the group level (i.e., $\mathrm{AD}-\mathrm{CN})$. 


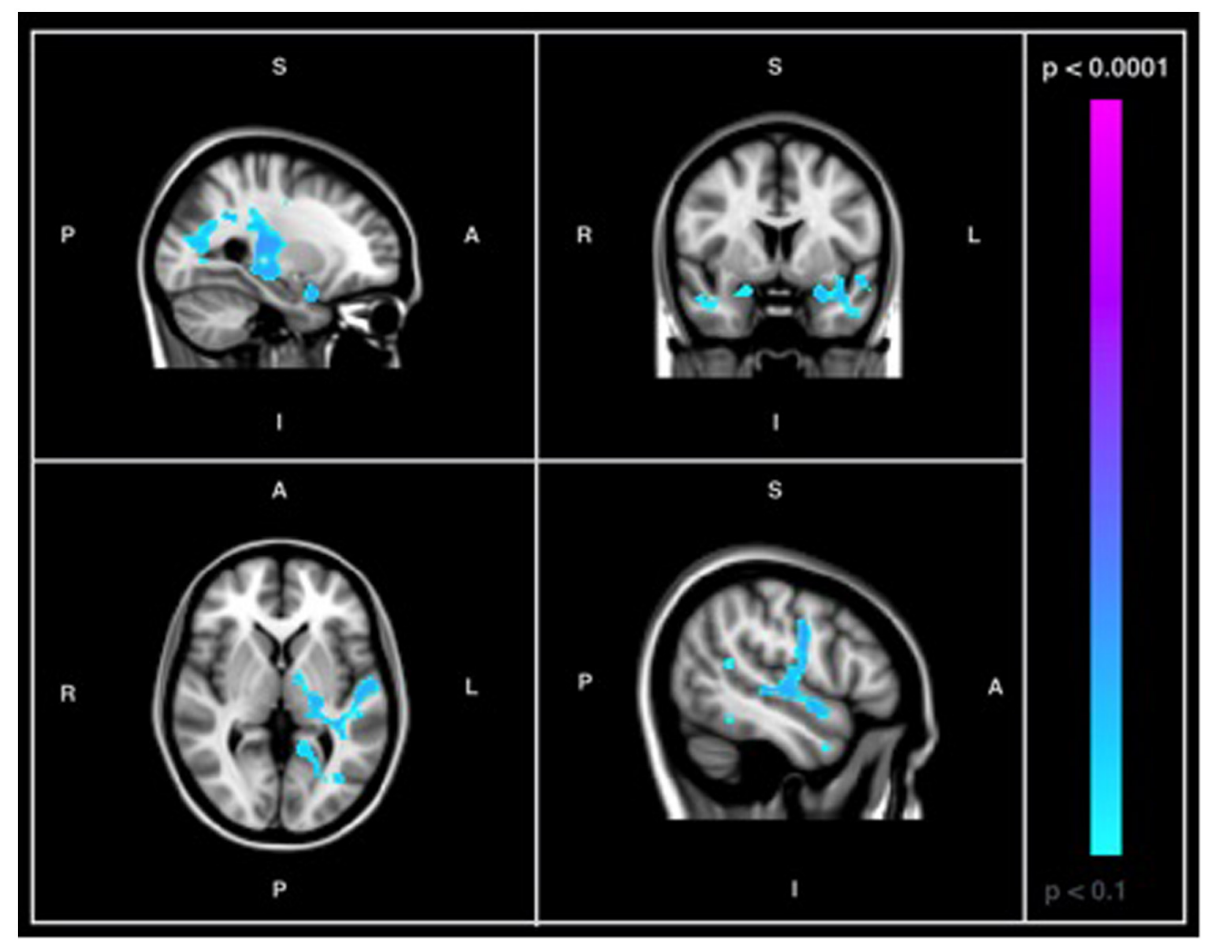

FIGURE 2 | Images showing GM and WM regions where $\mathrm{SD}_{B O L D}$ is negatively associated with ADNI-MEM scores in healthy controls ( $p<0.1$, corrected for multiple comparisons). Images on overlaid on T1-weighted MNI152_T1_2mm standard template provided by the Functional MRI of the Brain's Software Library.

Given the present finding of increased signal variability in patients with $\mathrm{AD}$ relative to healthy controls, an important question remains: what might be the underlying psychophysiological correlates driving the increased signal variability in $\mathrm{AD}$ ?

\section{$\mathrm{SD}_{\mathrm{BOLD}}$ and Its Association with Cognitive Function}

Central to the aforementioned compensation hypothesis, and reflected in previous findings of BOLD variability (e.g., Grady and Garrett, 2014), is the notion that $\mathrm{SD}_{\mathrm{BOLD}}$ may serve as a neuronal index of cognitive function. Therefore, the second objective of the current study was to determine whether measures of BOLD variability might be associated with measures of memory and executive function (ADNI-MEM and ADNI-EF). Contrary to our hypothesis, we did not find a significant relationship between $\mathrm{SD}_{\mathrm{BOLD}}$ and clinical test performance in patients with $\mathrm{AD}$ or healthy age-matched controls. Due to the preliminary nature of the present investigation, results were also examined with a more liberal threshold $(p<0.1)$. In doing so, an association was identified in the healthy control group, revealing a negative relationship between $\mathrm{SD}_{\mathrm{BOLD}}$ and composite memory scores. Specifically, lower memory scores were associated with greater $\mathrm{SD}_{\mathrm{BOLD}}$ in the healthy control group in the medial temporal lobe and adjacent structures (Table 4). No association was found between composite memory scores and $\mathrm{SD}_{\mathrm{BOLD}}$ in the $\mathrm{AD}$ group. Moreover, no association was identified between composite scores of executive function and $\mathrm{SD}_{\mathrm{BOLD}}$ in $\mathrm{AD}$ patients or healthy controls.

Though tentative interpretation is required, the trend toward a negative relationship identified in the healthy control group appears at odds with previous rsfMRI BOLD variability studies on healthy aging populations that have found that higher fluid intelligence and memory scores were linked to greater $\mathrm{SD}_{\mathrm{BOLD}}$ in diffuse cortical regions (Burzynska et al., 2015b). However, these findings are more consistent with those by Makedonov et al. (2016), who examined patients with AD from the ADNI database and found that greater WM BOLD fluctuations were associated with lower composite scores of memory function in patients with $\mathrm{AD}$, with no relationship identified between BOLD fluctuations and scores of executive function.

A number of factors may have contributed to the inconsistent association between BOLD variability and cognition observed in the present study. Notably, when Makedonov et al. (2016) included diagnostic status, glucose metabolism, and both whole brain and global nuisance signal regressors in a final omnibus model, composite memory scores were no longer found to be significantly associated with WM BOLD fluctuations. The authors postulate that WM BOLD fluctuations may therefore provide novel information about diagnostic status that is not captured by existing biomarkers, including cognition (Makedonov et al., 2016). Thus, it is possible that the trendlevel association between $\mathrm{SD}_{\mathrm{BOLD}}$ and memory observed in the control group merely reflects a corollary association between BOLD variability and another factor associated with memory 

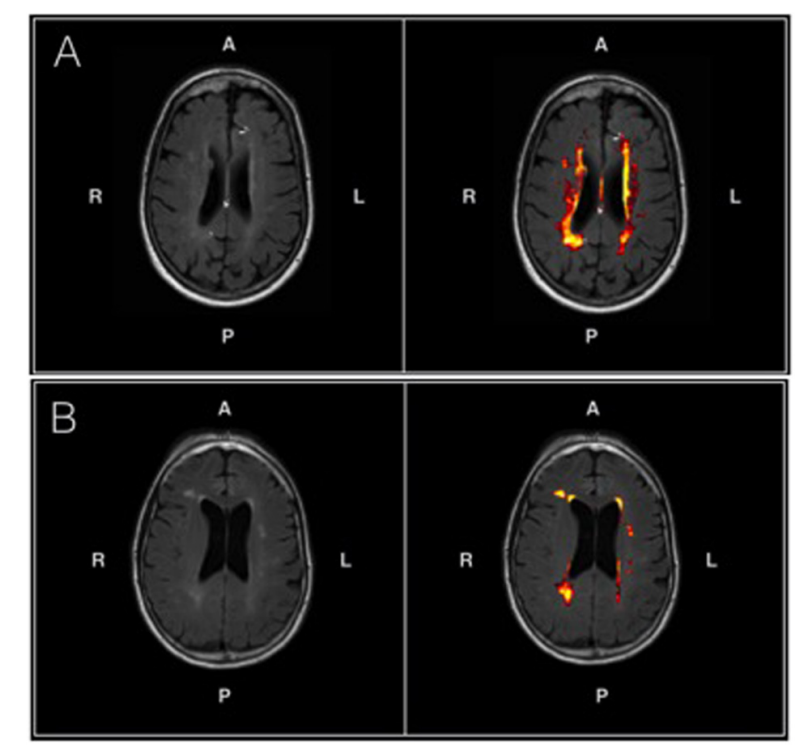

FIGURE 3 | (A) An axial T2-FLAIR image of a prototypical patient from the AD group (left) and associated probabilistic lesion volume map (right) generated by the LST-LPA. (B) An axial T2-FLAIR image of a prototypical patient from the healthy control group (left) and associated probabilistic lesion volume map (right) generated by the LST-LPA. Brighter (yellow) hues indicate a higher lesion probability in that area, with lower lesion probabilities indicated by darker (red) hues. Prototypical AD and healthy control participants were selected based on the proximity of their WM lesion burdens to their respective group means.

composite scores in normative aging, that is otherwise disrupted by the diffuse pathophysiological changes in AD.

Another possibility for the present findings relates to the previous discussion on neural compensation in aging. If $\mathrm{SD}_{\mathrm{BOLD}}$ indeed reflects compensatory activity, then it is reasonable to assume that there are likely limits to how effective that compensation can be. Indeed, it is possible that in $\mathrm{AD}$ such compensatory mechanisms are no longer effective, thereby rendering void any observable relationship between $\mathrm{SD}_{\mathrm{BOLD}}$ and memory or executive abilities in the $\mathrm{AD}$ group. In contrast, the associations observed in normal aging may reflect active residual compensation effects.

However, it is also notable that many of the GM regions in which we found BOLD variability to be negatively associated (at trend-level) with composite memory scores in healthy controls included critical structures of the MTL known to subserve memory function, including the amygdala, hippocampus and parahippocampal gyri (Scoville and Milner, 1957; Cohen and Eichenbaum, 1993; Squire et al., 2004). In early AD, memory disturbances are often the primary and most salient clinical concern (Sperling et al., 2010). Moreover, in these early stages of the disease process, neuron loss figures most prominently in these MTL structures (Bottino et al., 2002). In addition to structural changes, up-regulatory functional changes are also known to occur in MTL memory circuits in patients with AD and in persons with mild cognitive impairment (Dickerson and Sperling, 2008; Faraco et al., 2013). Thus, the present findings suggest that resting state $\mathrm{SD}$ BOLD may provide a novel
TABLE 5 | Brain regions showing a positive association between $\mathrm{SD}_{\mathrm{BOLD}}$ and white matter hyperintensity burden in the healthy control group $(p<0.1$, corrected for multiple comparisons)

\begin{tabular}{lcrcc}
\hline Brain region & Hemisphere & \multicolumn{3}{c}{ MNI coordinates } \\
\cline { 3 - 5 } & & $\mathbf{x}$ & $\mathbf{y}$ & $\mathbf{z}$ \\
\hline Gray matter & & & & \\
Parahippocampal gyrus & $\mathrm{R}$ & 26 & -32 & -14 \\
Central opercular cortex & $\mathrm{R}$ & 46 & -14 & 12 \\
Heschl's gyrus & $\mathrm{R}$ & 44 & -14 & 8 \\
Orbitofrontal cortex & $\mathrm{L}$ & -32 & 20 & -26 \\
White matter & & & & \\
Posterior corona radiata & $\mathrm{L}$ & -28 & -54 & 24 \\
\hline
\end{tabular}

Coordinates are shown in Montreal Neurological Institute (MNI) standard stereotaxic space.

method of characterizing changes in functional integrity in these regions.

In order to further elucidate the association between $\mathrm{SD}_{\mathrm{BOLD}}$ in behaviorally relevant regions of the MTLs and its association with pathological aging, there is a need for additional longitudinal studies examining $\mathrm{SD}_{\mathrm{BOLD}}$ and its association with specific measures of memory and cognition in prodromal aging groups with conversion to AD.

\section{SD $_{\text {BOLD }}$ and Its Association with Cerebrovascular Health}

Although cerebrovascular factors have previously been hypothesized to underlie BOLD fluctuation patterns in $\mathrm{AD}$ (Makedonov et al., 2016), this relationship had not been examined until the present study. Specifically, the third objective of the current study was to examine the association between GM and WM BOLD variability and neuroimaging markers of WM cerebrovascular burden. In light of the assertions by Makedonov et al. (2013, 2016), we hypothesized that there would be a positive association between resting-state BOLD variability and MRI-based measures of WM lesion load. When conventional thresholds were used, we did not find a significant association between total WMH burden and $\mathrm{SD}_{\mathrm{BOLD}}$ in patients with $\mathrm{AD}$ or healthy controls. However, as in the previous analysis, we also chose to examine more liberal thresholds in order to further explore any potential directionality in the data. In doing so, an association was again identified in the healthy control group, showing a positive association between WMH lesion burden and $\mathrm{SD}_{\mathrm{BOLD}}$. Specifically, participants with a higher WM lesion burden had greater $\mathrm{SD}_{\mathrm{BOLD}}$ in a set of highly localized brain regions, among which most prominently included the right parahippocampal gyrus and right temporal cortex (Figure 4).

Though tentative, the present results are in line with the initial findings by Makedonov et al. (2013), who discovered that WM rsfMRI BOLD fluctuations were increased in the WM of patients with cerebral small vessel disease and positively correlated with WMH volume. This increased BOLD temporal variance has been suggested to reflect greater pulsatility in vascular networks and small vessels as a result of reduced cerebrovascular 


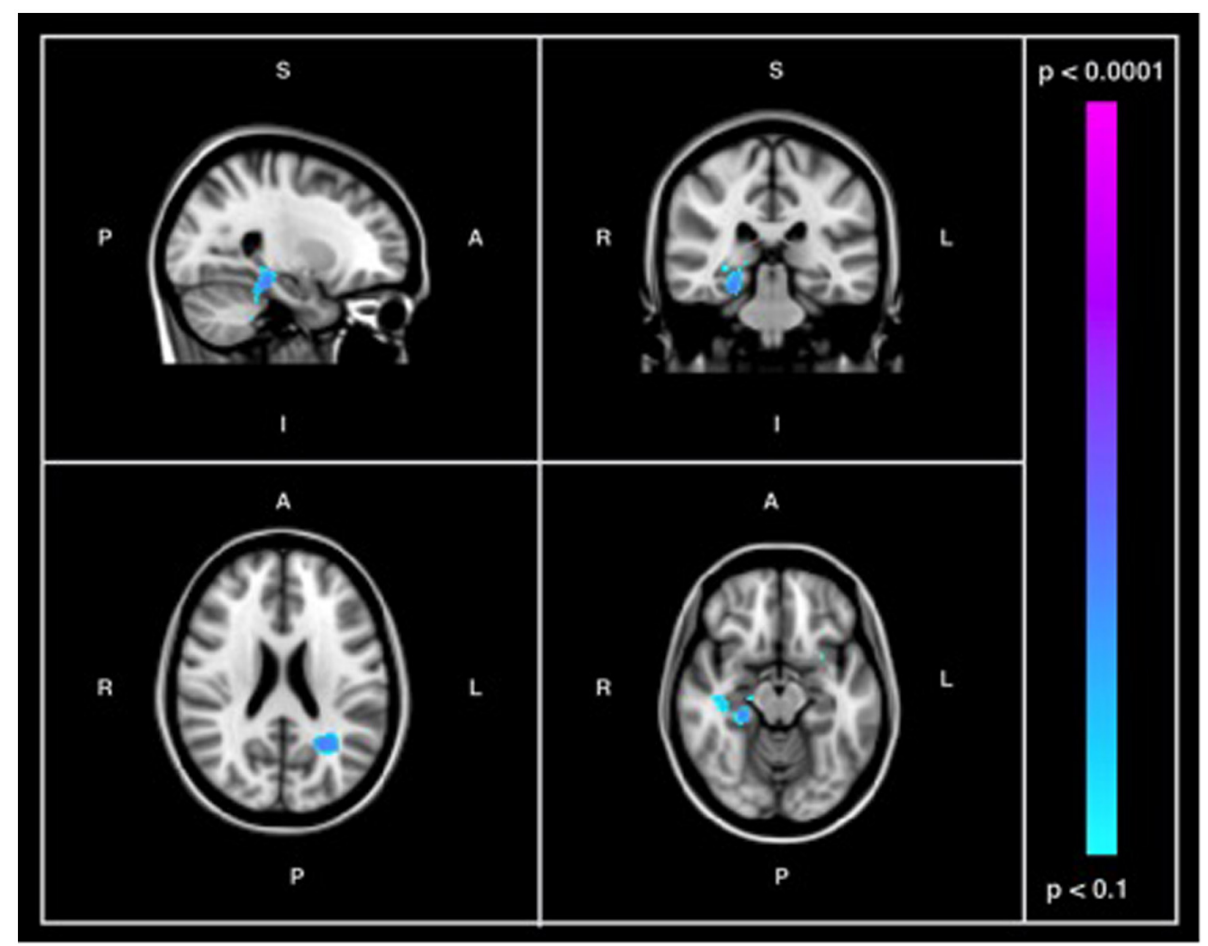

FIGURE 4 | Images showing GM and WM regions where $\mathrm{SD}_{B O L D}$ is positively associated with WMH burden in the healthy control group $(p<0.1$, corrected for multiple comparisons). Images on overlaid on T1-weighted MNI152_T1_2mm standard template provided by the Functional MRI of the Brain's Software Library.

TABLE 6 | Partial correlation coefficients $(r)$ for the relation between cognitive performance (ADNI-MEM and ADNI-EF) and WMH burden across groups, corrected for age and education.

\begin{tabular}{lcc}
\hline & WMH Burden Corr. (r) & Significance \\
\hline ADNI-MEM & -0.462 & $p=0.008$ \\
ADNI-EF & -0.428 & $p=0.015$ \\
\hline
\end{tabular}

compliance (Makedonov et al., 2013, 2016). Other studies have also lent support to this vascular interpretation in both clinically hypertensive patients and healthy aging populations (Kannurpatti and Biswal, 2008; Kannurpatti et al., 2011; Jahanian et al., 2014).

Though the current results do not appear to support an association between resting-state BOLD variability and $\mathrm{WMH}$ lesion burden in $\mathrm{AD}$, further considerations are warranted. Specifically, due to strict exclusion criteria necessitating a group of patients with $\mathrm{AD}$ without significant vascular comorbidity, the WHM lesion burden in ADNI appears relatively low (Ramirez et al., 2016), which may have contributed to underpowered effects. However, despite low WMH burden levels, there is evidence to suggest that they are non-trivial. For instance, Carmichael et al. (2010) found that WMH volumes at baseline predicted 1-year global cognitive decline in a sample of over 800 participants from the ADNI database. Moreover, consistent with this existing literature, results from our data suggest that greater vascular insult is uniformly associated with lower memory and executive functioning across the sample, even after correcting for age and education. This again echoes the need to re-examine the neurophysiological correlates of $\mathrm{SD}_{\mathrm{BOLD}}$ from a longitudinal perspective.

Moreover, although the current study detected a trending relationship between vascular burden and BOLD signal variability in healthy controls, but not in patients with $A D$, the association between vascular risk factors and $\mathrm{AD}$ is one that is well established (O'Brien and Markus, 2014; Cai et al., 2015). Indeed, vascular risk factors, including hypertension and cholesterolemia, have been found to be significant risk factors for $\mathrm{AD}$-specific neuropathology, including neuritic plaques and neurofibrillary tangles (Petrovitch et al., 2000; O'Brien and Markus, 2014). Interestingly, and in accordance with the results of the present study, a meta-analysis by Debette and Markus (2010) also found that WMHs, which are known markers of cerebrovascular outcomes (Debette and Markus, 2010; Chutinet and Rost, 2014), tended to be associated with an increased risk of dementia and AD in healthy populations, but not in patients who already exhibit cognitive impairment. In light of this evidence, examination of $\mathrm{SD}_{\mathrm{BOLD}}$ as a non-invasive biomarker for underlying cerebrovascular risk factors in aging is one that directly contributes to the ultimate goal of improving the early identification of $\mathrm{AD}$. For these reasons, further exploration of the link between

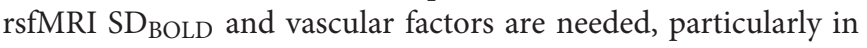
prodromal aging groups and with multimodal measures that may more comprehensively capture underlying cerebrovascular risk. 


\section{Study Limitations}

As discussed previously, one primary limitation of the current study relates to the diverse conceptualizations of fMRI BOLD variability, which have varied considerably across studies, making it challenging to systematically compare findings. Specifically, several different variations of 'BOLD variability' measures have been described (e.g., amplitude, variance, standard deviation, mean squared successive difference; for a review, see Garrett et al., 2013b), with considerable range in the methodology used to derive them. As argued by Garrett et al. (2013b), a significant barrier to adopting this novel imaging method on a larger scale lies in the lack of signal variability estimation tools in major neuroimaging software packages. Though some efforts have been made by Garrett et al. (2013b) to systematize the analysis of signal variability, implementation of BOLD variability as a novel imaging biomarker for $\mathrm{AD}$ will require increased efforts toward methodological standardization.

Another potential limitation of the current study is that only WMH burden was examined as a proxy for cerebrovascular status across groups. While WMHs are believed to be an indicator of cerebral small vessel disease (Mok and Kim, 2015) and cerebral arterial stiffness has found to be correlated with WHM lesion volume (Kidwell et al., 2001), they remain nonspecific proxy measures. Future work should include more direct measures of cerebrovascular reactivity to re-examine the hypothesis that BOLD variability in neurodegenerative disease may reflect underlying cerebrovascular factors. Moreover, due to the strict exclusion criteria and, thus, the relatively low WMH lesion burden in the ADNI sample, future studies should examine more ecologically valid patient samples of $\mathrm{AD}$ with mixed vascular pathologies (Ramirez et al., 2016).

Finally, the sample size of the current study is small and limited to a single time point. To acquire a better understanding of BOLD variability and its association with $\mathrm{AD}$ pathology over time, future studies should examine larger samples at multiple time points of disease progression, which can be done in an ongoing manner as the ADNI dataset grows over time.

\section{CONCLUSION}

Increasingly, $\mathrm{AD}$ has become an urgent public health concern, rendering critical the need to improve upon its early identification, so that disease-delaying treatments may be implemented as soon as they become available. In support of this goal, the current study examined a novel approach to the analysis of rsfMRI data. Though, traditionally, fMRI investigations have based findings on patterns of mean brain activity, moment-to-moment variability in the BOLD signal may provide new information on disorders of aging and their associated clinical correlates (Garrett et al., 2013b). The current study found increased $\mathrm{SD}_{\mathrm{BOLD}}$ in patients with $\mathrm{AD}$ relative to healthy controls, with trends suggesting an association between $\mathrm{SD}_{\mathrm{BOLD}}$ and both memory performance and $\mathrm{WMH}$ lesion burden in the control group. To further examine the clinical utility of this novel imaging parameter, future work should focus on longitudinal studies of SDBOLD and its association with more comprehensive clinical and cerebrovascular data in both $\mathrm{AD}$ and prodromal aging.

\section{AUTHOR CONTRIBUTIONS}

VS was responsible for data analyses and drafting the manuscript. EM was responsible for designing the analyses. JF and LR were involved in interpretation of the results and critical revisions of the manuscript. JG supervised the project and was involved in data analyses, interpretation and critical revisions of the manuscript.

\section{FUNDING}

Funding for this project was made possible by generous support from the Canadian Institutes of Health Research (CIHR) and the Fonds de recherche du Québec - Santé (FRQS). Data collection and sharing for this project was funded by the Alzheimer's Disease Neuroimaging Initiative (ADNI) (National Institutes of Health Grant U01 AG024904) and DOD ADNI (Department of Defense award number W81XWH-12-2- 0012). ADNI is funded by the National Institute on Aging, the National Institute of Biomedical Imaging and Bioengineering, and through contributions from the following: AbbVie, Alzheimer's Association; Alzheimer's Drug Discovery Foundation; Araclon Biotech; BioClinica, Inc.; Biogen; BristolMyers Squibb Company; CereSpir, Inc.; Cogstate; Eisai Inc.; Elan Pharmaceuticals, Inc.; Eli Lilly and Company; EuroImmun; F. Hoffmann-La Roche Ltd and its affiliated company Genentech, Inc.; Fujirebio; GE Healthcare; IXICO Ltd.; Janssen Alzheimer Immunotherapy Research \& Development, LLC.; Johnson \& Johnson Pharmaceutical Research \& Development LLC.; Lumosity; Lundbeck; Merck \& Co., Inc.; Meso Scale Diagnostics, LLC.; NeuroRx Research; Neurotrack Technologies; Novartis Pharmaceuticals Corporation; Pfizer Inc.; Piramal Imaging; Servier; Takeda Pharmaceutical Company; and Transition Therapeutics. The Canadian Institutes of Health Research is providing funds to support ADNI clinical sites in Canada. Private sector contributions are facilitated by the Foundation for the National Institutes of Health (www.fnih.org). The grantee organization is the Northern California Institute for Research and Education, and the study is coordinated by the Alzheimer's Therapeutic Research Institute at the University of Southern California. ADNI data are disseminated by the Laboratory for Neuro Imaging at the University of Southern California.

\section{ACKNOWLEDGMENTS}

The study described herein, and all of its contents, first appeared in an author's thesis in partial fulfillment of a Master of Science (Scarapicchia, 2017). 


\section{REFERENCES}

Alzheimer's Association (2016). 2016 Alzheimer's disease facts and figures. Alzheimer's Dement. 12, 459-509. doi: 10.1016/j.jalz.2016.03.001

Alzheimer's Disease Neuroimaging Initiative (2008). ADNI-2 Procedures Manual. Available at: https://adni.loni.usc.edu/wp-content/uploads/2008/07/adni2procedures-manual.pdf.

Baudic, S., Dalla Barba, G., Thibaudet, M. C., Smagghe, A., Remy, P., and Traykov, L. (2006). Executive function deficits in early Alzheimer's disease and their relations with episodic memory. Arch. Clin. Neuropsychol. 21, 15-21. doi: 10.1016/j.acn.2005.07.002

Bottino, C. M. C., Castro, C. C., Gomes, R. L. E., Buchpiguel, C. A., Marchetti, R. L., and Neto, M. R. L. (2002). Volumetric MRI measurements can differentiate Alzheimer's disease, mild cognitive impairment, and normal aging. Int. Psychogeriatr. 14, 59-72. doi: 10.1017/S104161020200 8281

Burzynska, A. Z., Wong, C. N., Voss, M. W., Cooke, G. E., Gothe, N. P., Fanning, J., et al. (2015a). Physical activity is linked to greater moment-to- moment variability in spontaneous brain activity in older adults. PLoS One 10:e134819. doi: 10.1371/journal.pone.0134819

Burzynska, A. Z., Wong, C. N., Voss, M. W., Cooke, G. E., McAuley, E., and Kramer, A. F. (2015b). White matter integrity supports BOLD signal variability and cognitive performance in the aging human brain. PLoS One 10:e0120315. doi: 10.1371/journal.pone.0120315

Cai, Z., Wang, C., He, W., Tu, H., Tang, Z., Xiao, M., et al. (2015). Cerebral small vessel disease and Alzheimer's disease. Clin. Interv. Aging 10, 1695-1704. doi: 10.2147/CIA.S90871

Carmichael, O., Schwarz, C., Drucker, D., Fletcher, E., Harvey, D., Beckett, L., et al. (2010). Longitudinal changes in white matter disease and cognition in the first year of the Alzheimer disease neuroimaging initiative. Arch. Neurol. 67, 1370-1378. doi: 10.1001/archneurol.2010.284

Cash, D. M., Rohrer, J. D., Ryan, N. S., Ourselin, S., and Fox, N. C. (2014). Imaging endpoints for clinical trials in Alzheimer's disease. Alzheimer's Res. Ther. 6:87. doi: 10.1186/s13195-014-0087-9

Chutinet, A., and Rost, N. S. (2014). White matter disease as a biomarker for longterm cerebrovascular disease and dementia. Curr. Treat. Options Cardiovasc. Med. 16:292. doi: 10.1007/s11936-013-0292-z

Cohen, N. J., and Eichenbaum, H. (1993). Memory, Amnesia, and the Hippocampal System. Cambridge, MA: MIT Press.

Crane, P. K., Carle, A., Gibbons, L. E., Insel, P., Mackin, R. S., Gross, A., et al. (2012). Development and assessment of a composite score for memory in the Alzheimer's disease neuroimaging initiative (ADNI). Brain Imaging Behav. 6, 502-516. doi: 10.1007/s11682-012-9186-z

Debette, S., and Markus, H. S. (2010). The clinical importance of white matter hyperintensities on brain magnetic resonance imaging: systematic review and meta-analysis. Br. Med. J. 341:c3666. doi: 10.1136/bmj.c3666

Deco, G., Jirsa, V. K., and McIntosh, A. R. (2011). Emerging concepts for the dynamical organization of resting-state activity in the brain. Nat. Rev. Neurosci. 12, 43-56. doi: 10.1038/nrn2961

Desjardins, A. E., Kiehl, K. A., and Liddle, P. F. (2001). Removal of confounding effects of global signal in functional MRI analyses. NeuroImage 13, 751-758. doi: 10.1038/nrn2961

Dickerson, B. C., and Sperling, R. A. (2008). Functional abnormalities of the medial temporal lobe memory system in mild cognitive impairment and Alzheimer's disease: insights from functional MRI studies. Neuropsychologia 46, 1624-1635. doi: 10.1016/j.neuropsychologia.2007.11.030

Faisal, A. A., Selen, L. P. J., and Wolpert, D. M. (2008). Noise in the nervous system. Nat. Rev. Neurosci. 9, 292-303. doi: 10.1016/j.neuropsychologia.2007.11.030

Faraco, C. C., Puente, A. N., Brown, C., Terry, D. P., and Miller, L. S. (2013). Lateral temporal hyper-activation as a novel biomarker of mild cognitive impairment. Neuropsychologia 51, 2281-2293. doi: 10.1016/j.neuropsychologia.2013.07.023

Fox, M. D., and Greicius, M. (2010). Clinical applications of resting state functional connectivity. Front. Syst. Neurosci. 4:19. doi: 10.3389/fnsys.2010.00019

Fox, M. D., Zhang, D., Snyder, A. Z., and Raichle, M. E. (2009). The global signal and observed anticorrelated resting state brain networks. J. Neurophysiol. 101, 3270-3283. doi: 10.1152/jn.90777.2008
Garrett, D., Kovacevic, N., McIntosh, A., and Grady, C. (2011). The importance of being variable. J. Neurosci. 31, 4496-4503. doi: 10.1523/JNEUROSCI.5641-10. 2011

Garrett, D. D., Kovacevic, N., McIntosh, A. R., and Grady, C. L. (2010). Blood oxygen level-dependent signal variability is more than just noise. J. Neurosci. 30, 4914-4921. doi: 10.1523/JNEUROSCI.5166-09.2010

Garrett, D. D., Kovacevic, N., McIntosh, A. R., and Grady, C. L. (2013a). The modulation of BOLD variability between cognitive states varies by age and processing speed. Cereb. Cortex 23, 684-693. doi: 10.1093/cercor/bhs055

Garrett, D. D., McIntosh, A. R., and Grady, C. L. (2014). Brain signal variability is parametrically modifiable. Cereb. Cortex 24, 2931-2940. doi: 10.1093/cercor/ bht150

Garrett, D. D., Samanez-Larkin, G. R., MacDonald, S. W. S., Lindenberger, U., McIntosh, A. R., and Grady, C. L. (2013b). Moment-to-moment brain signal variability: a next frontier in human brain mapping? Neurosci. Biobehav. Rev. 37, 610-624. doi: 10.1016/j.neubiorev.2013.02.015

Gauthier, C. J., Madjar, C., Desjardins-Crépeau, L., Bellec, P., Bherer, L., and Hoge, R. D. (2013). Age dependence of hemodynamic response characteristics in human functional magnetic resonance imaging. Neurobiol. Aging 34, 1469-1485. doi: 10.1016/j.neurobiolaging.2012.11.002

Gibbons, L. E., Carle, A. C., Mackin, R. S., Harvey, D., Mukherjee, S., Insel, P., et al. (2012). A composite score for executive functioning, validated in Alzheimer's Disease neuroimaging initiative (ADNI) participants with baseline mild cognitive impairment. Brain Imaging Behav. 6, 517-527. doi: 10.1016/j. neurobiolaging.2012.11.002

Grady, C. L., and Garrett, D. D. (2014). Understanding variability in the BOLD signal and why it matters for aging. Brain Imaging Behav. 8, 274-283. doi: 10.1007/s1 1682-013-9253-0

Han, Y., Wang, J., Zhao, Z., Min, B., Lu, J., Li, K., et al. (2011). Frequencydependent changes in the amplitude of low-frequency fluctuations in amnestic mild cognitive impairment: a resting-state fMRI study. Neuroimage 55, 287-295. doi: 10.1016/j.neuroimage.2010.11.059

Jack, C. R., Bernstein, M. A., Fox, N. C., Thompson, P., Alexander, G., Harvey, D., et al. (2008). The Alzheimer's disease neuroimaging initiative (ADNI): MRI methods. J. Magn. Reson. Imaging? 27, 685-691. doi: 10.1002/jmri.21049

Jahanian, H., Ni, W. W., Christen, T., Moseley, M. E., Tamura, M. K., and Zaharchuk, G. (2014). Spontaneous BOLD signal fluctuations in young healthy subjects and elderly patients with chronic kidney disease. PLoS One 9:e92539. doi: 10.1371/journal.pone.0092539

Kannurpatti, S. S., and Biswal, B. B. (2008). Detection and scaling of taskinduced $\mathrm{fMRI}$ - BOLD response using resting state fluctuations. Neuroimage 40, 1567-1574. doi: 10.1016/j.neuroimage.2007.09.040

Kannurpatti, S. S., Motes, M. A., Rypma, B., and Biswal, B. B. (2011). Increasing measurement accuracy of age-related BOLD signal change: minimizing vascular contributions by resting-state-fluctuation-of-amplitude scaling. Hum. Brain Mapp. 32, 1125-1140. doi: 10.1002/hbm.21097

Kidwell, C. S., El-Saden, S., Livshits, Z., Martin, N. A., Glenn, T. C., and Saver, J. L. (2001). Transcranial doppler pulsatility indices as a measure of diffuse smallvessel disease. J. Neuroimaging 11, 229-235. doi: 10.1002/hbm.21097

Kielar, A., Deschamps, T., Chu, R. K., Jokel, R., Khatamian, Y. B., Chen, J. J., et al. (2016). Identifying dysfunctional cortex: dissociable effects of stroke and aging on resting state dynamics in MEG and fMRI. Front. Aging Neurosci. 8:40. doi: 10.3389/fnagi.2016.00040

Liu, C. Y., Krishnan, A. P., Yan, L., Smith, R. X., Kilroy, E., Alger, J. R., et al. (2013). Complexity and synchronicity of resting state blood oxygenation leveldependent (BOLD) functional MRI in normal aging and cognitive decline. J. Magn. Reson. Imaging? 38, 36-45. doi: 10.1002/jmri.23961

Liu, X., Wang, S., Zhang, X., Wang, Z., Tian, X., and He, Y. (2014). Abnormal amplitude of low-frequency fluctuations of intrinsic brain activity in Alzheimer's disease. J. Alzheimer's Dis. 40, 387-397. doi: 10.3233/JAD-131322

Livingston, G., Sommerlad, A., Orgeta, V., Costafreda, S. G., Huntley, J., Ames, D., et al. (2017). Dementia prevention, intervention, and care. Lancet 390, 2673-2734. doi: 10.1016/S0140-6736(17)31363-6

Macey, P. M., Macey, K. E., Kumar, R., and Harper, R. M. (2004). A method for removal of global effects from fMRI time series. NeuroImage 22, 360-366. doi: 10.1016/j.neuroimage.2003.12.042 
Makedonov, I., Black, S. E., and MacIntosh, B. J. (2013). BOLD fMRI in the white matter as a marker of aging and small vessel disease. PLoS One 8:e67652. doi: 10.1371/journal.pone.0067652

Makedonov, I., Chen, J. J., Masellis, M., and MacIntosh, B. J. (2016). Physiological fluctuations in white matter are increased in Alzheimer's disease and correlate with neuroimaging and cognitive biomarkers. Neurobiol. Aging 37, 12-18. doi: 10.1016/j.neurobiolaging.2015.09.010

McKhann, G., Drachman, D., Folstein, M., Katzman, R., Price, D., and Stadlan, E. M. (1984). Clinical diagnosis of Alzheimer's disease: report of the NINCDSADRDA Work group* under the auspices of department of health and human services task force on Alzheimer's Disease. Neurology 34, 939-944. doi: 10.1212/ WNL.34.7.939

Mok, V., and Kim, J. S. (2015). Prevention and management of cerebral small vessel disease. J. Stroke 17, 111-122. doi: 10.5853/jos.2015.17.2.111

Mueller, S., Keeser, D., Reiser, M. F., Teipel, S., and Meindl, T. (2012). Functional and structural MR imaging in neuropsychiatric disorders, Part 1: imaging techniques and their application in mild cognitive impairment and Alzheimer disease. Am. J. Neuroradiol. 33, 1845-1850. doi: 10.3174/ajnr.A2799

Nomi, J. S., Bolt, T. S., Ezie, C. C., Uddin, L. Q., and Heller, A. S. (2017). Moment-to- moment BOLD signal variability reflects regional changes in neural flexibility across the lifespan. J. Neurosci. 37, 5539-5548. doi: 10.1523/ JNEUROSCI.3408-16.2017

O'Brien, J. T., and Markus, H. S. (2014). Vascular risk factors and Alzheimer's disease. BMC Med. 12:218. doi: 10.1186/s12916-014-0218-y

Petracca, M., Saiote, C., Bender, H. A., Arias, F., Farrell, C., Magioncalda, P., et al. (2017). Synchronization and variability imbalance underlie cognitive impairment in primary-progressive multiple sclerosis. Sci. Rep. 7:46411. doi: $10.1038 /$ srep46411

Petrovitch, H., White, L. R., Izmirilian, G., Ross, G. W., Havlik, R. J., and Markesbery, W. (2000). Midlife blood pressure and neuritic plaques, neurofibrillary tangles, and brain weight at death: the HAAS. Honolulu-Asia aging study. Neurobiol. Aging 21, 57-62. doi: 10.1016/S0197-4580(00)00106-8

Ramirez, J., McNeely, A. A., Scott, C. J., Masellis, M., Black, S. E., and Alzheimer's Disease Neuroimaging Initiative. (2016). White matter hyperintensity burden in elderly cohort studies: the Sunnybrook dementia study, Alzheimer's disease neuroimaging initiative, and three-city study. Alzheimer's Dement. 12, 203-210. doi: $10.1016 /$ j.jalz.2015.06.1886

Reuter-Lorenz, P. A., and Park, D. C. (2010). Human neuroscience and the aging mind: a new look at old problems. J. Gerontol. B Psychol. Sci. Soc. Sci. 65B, 405-415. doi: 10.1093/geronb/gbq035

Scarapicchia, V. (2017). Resting State Bold Variability in Alzheimer's Disease: A Marker of Cognitive Decline or Cerebrovascular Status. Ph.D. thesis, University of Victoria, Victoria, BC.

Scheltens, P., Blennow, K., Breteler, M. M., de Strooper, B., Frisoni, G. B., Salloway, S., et al. (2016). Alzheimer's disease. Lancet 388, 505-517. doi: 10.1016/S0140-6736(15)01124-1

Schmidt, P. (2017). Bayesian Inference for Structured Additive Regression Models for Large-Scale Problems with Applications to Medical Imaging. Doctoral Dissertation, Ludwig-Maximilians-Universität München, Munich.

Schmidt, P., Gaser, C., Arsic, M., Buck, D., Förschler, A., Berthele, A., et al. (2012). An automated tool for detection of FLAIR-hyperintense white-matter lesions in multiple sclerosis. Neuroimage 59, 3774-3783. doi: 10.1016/j.neuroimage.2011. 11.032

Scoville, W. B., and Milner, B. (1957). Loss of recent memory after bilateral hippocampal lesions. J. Neurol. Neurosurg. Psychiatry 20, 11-21. doi: 10.1136/ jnnp.20.1.11
Smith, S. M. (2002). Fast robust automated brain extraction. Hum. Brain Mapp. 17, 143-155. doi: 10.1002/hbm.10062

Smith, S. M., Jenkinson, M., Woolrich, M. W., Beckmann, C. F., Behrens, T. E. J., Johansen-Berg, H., et al. (2004). Advances in functional and structural MR image analysis and implementation as FSL. NeuroImage 23(Suppl. 1), S208-S219. doi: 10.1016/j.neuroimage.2004.07.051

Sperling, R. A., Dickerson, B. C., Pihlajamaki, M., Vannini, P., LaViolette, P. S., Vitolo, O. V., et al. (2010). Functional alterations in memory networks in early Alzheimer's disease. Neuromolecular Med. 12, 27-43. doi: 10.1007/s12017-0098109-7

Squire, L. R., Stark, C. E. L., and Clark, R. E. (2004). The medial temporal lobe. Annu. Rev. Neurosci. 27, 279-306. doi: 10.1146/annurev.neuro.27.070203. 144130

Stein, R. B., Gossen, E. R., and Jones, K. E. (2005). Neuronal variability: noise or part of the signal? Nat. Rev. Neurosci. 6, 389-397. doi: 10.1038/ nrn 1668

Wilkinson, D. (2012). A review of the effects of memantine on clinical progression in Alzheimer's disease. Int. J. Geriatr. Psychiatry 27, 769-776. doi: 10.1002/gps. 2788

Winblad, B., Amouyel, P., Andrieu, S., Ballard, C., Brayne, C., Brodaty, H., et al. (2016). Defeating Alzheimer's disease and other dementias: a priority for European science and society. Lancet Neurol. 15, 455-532. doi: 10.1016/S14744422(16)00062-4

Winkler, A. M., Ridgway, G. R., Webster, M. A., Smith, S. M., and Nichols, T. E. (2014). Permutation inference for the general linear model. Neuroimage 92, 381-397. doi: 10.1016/j.neuroimage.2014.01.060

Xi, Q., Zhao, X., Wang, P., Guo, Q., Jiang, H., Cao, X., et al. (2012). Spontaneous brain activity in mild cognitive impairment revealed by amplitude of lowfrequency fluctuation analysis: a resting-state fMRI study. Radiol. Med. 117, 865-871. doi: 10.1007/s11547-011-0780-8

Zhang, Y., Brady, M., and Smith, S. (2001). Segmentation of brain MR images through a hidden Markov random field model and the expectationmaximization algorithm. IEEE Trans. Med. Imaging 20, 45-57. doi: 10.1109/ 42.906424

Zhao, Z. L., Fan, F. M., Lu, J., Li, H. J., Jia, L. F., Han, Y., et al. (2015). Changes of gray matter volume and amplitude of low-frequency oscillations in amnestic MCI: an integrative multi-modal MRI study. Acta Radiol. 56, 614-621. doi: 10.1177/0284185114533329

Zöller, D., Schaer, M., Scariati, E., Padula, M. C., Eliez, S., and Van De Ville, D. (2017). Disentangling resting-state BOLD variability and PCC functional connectivity in 22q11. 2 deletion syndrome. NeuroImage 149, 85-97. doi: 10.1016/j.neuroimage.2017.01.064

Conflict of Interest Statement: The authors declare that the research was conducted in the absence of any commercial or financial relationships that could be construed as a potential conflict of interest.

The reviewer RR and the handling Editor declared their shared affiliation.

Copyright (c) 2018 Scarapicchia, Mazerolle, Fisk, Ritchie and Gawryluk. This is an open-access article distributed under the terms of the Creative Commons Attribution License (CC BY). The use, distribution or reproduction in other forums is permitted, provided the original author(s) and the copyright owner are credited and that the original publication in this journal is cited, in accordance with accepted academic practice. No use, distribution or reproduction is permitted which does not comply with these terms. 